\title{
Transgender Women with Severe Mental Illness and Homelessness: A Retrospective Case Series
}

\author{
Rafael Fernández García-Andrade1,2,3,4*, Beatriz Serván Rendón-Luna², Virginia Vidal Martínez \\ Elena Medina Téllez De Meneses ${ }^{1,6}$, Blanca Reneses Prieto²,3,4
}

\author{
${ }^{1}$ Mental Health Street Team of Madrid's “Programme for the Psychiatric Care of the Homeless Mentally Ill”, Madrid, Spain \\ ${ }^{2}$ Institute of Psychiatry and Mental Health of the Clínico San Carlos Hospital, Madrid, Spain \\ ${ }^{3}$ Health Research Institute of the Hospital Clínico San Carlos (IdISSC), Madrid, Spain \\ ${ }^{4}$ Department of Psychiatry, Faculty of Medicine, Complutense University, Madrid, Spain \\ ${ }^{5}$ Alcorcón University Hospital Foundation, Madrid, Spain \\ ${ }^{6} \mathrm{La}$ Paz University Hospital, Madrid, Spain \\ Email: ^rfgarciaandrade@salud.madrid.org, *primerosepisodios@hotmail.com
}

How to cite this paper: García-Andrade, R.F., Rendón-Luna, B.S., Martínez, V.V., De Meneses, E.M.T. and Prieto, B.R. (2019) Transgender Women with Severe Mental Illness and Homelessness: A Retrospective Case Series. Open Journal of Social Sciences, 7, 405-411.

https://doi.org/10.4236/jss.2019.78030

Received: June 28, 2019

Accepted: August 26, 2019

Published: August 29, 2019

Copyright $\odot 2019$ by author(s) and Scientific Research Publishing Inc. This work is licensed under the Creative Commons Attribution International License (CC BY 4.0).

http://creativecommons.org/licenses/by/4.0/

\begin{abstract}
Transgender women (TW) with Severe Mental Illness (SMI) are one of the most vulnerable and most difficult to engage in order to receive medical attention population, raising major challenges in their treatment of adverse health conditions. A retrospective chart review was carried out to identify TW with SMI attended by the "Programme for the Psychiatric Care of the Homeless Mentally Ill” in Spain from June 2015 to June 2018. During the study period, 235 patients with SMI received psychiatric care, of which only 3 (1.3\%) were TW. Sociodemographic and clinical variables of these patients are described. We conclude that TW with SMI suffer significant levels of discrimination, stigma, and physical violence. This stigma plays an important role in limiting the opportunities and access to resources in a number of critical domains (e.g., medical care, disability certificate, accommodation in shelters) while continuously having a detrimental effect on their mental health. Forced migration in TW with SMI, must be specially considered. In addition homelessness and social exclusion are structural risk factors for HIV in TW with SMI. Social, health and psychiatric care may moderate the effect of stressors on mental health, reduce social isolation, and build social networks.
\end{abstract}

\section{Keywords}

LGTB, Transgender Women, Mental Illness, Homeless Population, Homelessness 


\section{Introduction}

Transgender women (TW) face many challenges in society including accessing and using healthcare systems. Little is known about the specific mental health service needs and concerns of TW in this regard. Studies investigating the prevalence of psychiatric disorders among transgender individuals have identified elevated rates of psychopathology [1]. Research has also provided conflicting psychiatric outcomes following gender-confirming medical interventions [2]. TW with Severe Mental Illness (SMI) are one of the most vulnerable and most difficult to engage in order to receive medical attention population, raising major challenges in their treatment of adverse health conditions [3] [4]. The criterion for designating an individual as having an SMI is established by the United States' National Institute of Mental Health (NIMH), [5] which defines this social group as: "A group of heterogeneous individuals, who suffer from severe psychiatric illnesses, identified by mental illnesses of prolonged duration, which involve a variable degree of disability and social dysfunction, and which have to be attended to with various social and health resources in the psychiatric and social health care network".

The main objective of the study was to evaluate the sociodemographic and clinical profile of TW with SMI attended by the "Programme for the Psychiatric Care of the Homeless Mentally Ill" in Spain. The criterion for designating an individual as a homeless is established by the European Federation of National Organisations Working with the Homeless (FEANTSA) based on ETHOS (European Typology of Homelessness and Housing Exclusion) [5]. It states the following: "People who cannot access or retain adequate accommodation that is adapted to their personal situation, that is permanent, and that provides a stable living environment, either due to economic reasons or other social barriers, or because they have personal difficulties with leading an autonomous lifestyle".

\section{Materials and Methods}

This observational naturalistic study was done in the Mental Health Street Team of Madrid's "Programme for the Psychiatric Care of the Homeless Mentally Ill" in Spain. The abovementioned Programme provides social, health and psychiatric care for all adult homeless people in the municipality of Madrid (Spain) with SMI, and who for various reasons are not being monitored by the standard mental health network [3] [4]. We followed the criteria of the FEANTSA based on the ETHOS to categorise a person as homeless [6]. We used the criteria of NIMH to define SMI [5].

The sample study was selected from a total of 235 patients who had completed the Baseline Assessment Protocol (BAP), which is given to all subjects when they are included in the above mentioned Programme, and includes the following socio-demographic and clinical variables: year of birth gender, nationality, place of origin, type of living environment before homelessness, years of evolution of homelessness, typology of homelessness (according to ETHOS), educational lev- 
el reached, best profession reached, labor situation, income sources, health coverage, disability certificate, civil incapacity, victim of violence, co-morbidity with a history of substance use, Comorbidity with HIV, co-morbidity with severe somatic disease, main psychiatric diagnosis (according to ICD-10), years of evolution of the mental illness, form of evolution of the mental illness, previous contact with mental health services, previous psychiatric hospitalisation, disease awareness, attitude towards pharmacological treatment, severity of mental illness.

For this study, the BAP of the Programme was used to identify the transgender patients. At the first step, the BAP for the period June 2015-June 2018 was screened to identify all the transgender patients who received health and psychiatric care in the Programme. All patients were assessed with the variables present in the BAP, which includes extensive information on sociodemographic and clinical variables. Only TW cases were taken up for the study. For each subject, an anonymized form was filled in by each patient (generating an anonymized database). This study was performed with the approval of the Clinical Research Ethics Committee of Madrid's Clínico San Carlos, in compliance with all the requirements of the Declaration of Helsinki, and Spanish legislation on data protection. Descriptive statistics were used to analyze the data. SPSS version 14 was used for the analysis.

\section{Results}

During the study period, 235 patients received health and psychiatric care, of which only 3 (1.3\%) were TW. These patients (age mean 37.3 years) formed the study sample.

Sociodemographic and clinical variables of the patients included in the study are provided in Table 1.

\section{Discussion}

The present study showed that in a span of 3 years in the "Programme for the Psychiatric Care of the Homeless Mentally Ill", only 1.3\% patients were TW. However, it is a high percentage if we compare it with previous studies regarding the prevalence of homeless transgender people in an emergency hospital population in USA, which was $0.07 \%$ [9]. This could be explained because TW suffer behaviors such as labeling, separation, awareness of stereotypes and prejudices and discrimination. This stigma, along with SMI, is an important factor that prevents TW with SMI from seeking help. In addition, this stigma plays an important role in limiting the opportunities and access to resources of TW in several critical domains (e.g., medical care), while continuously having a detrimental effect on their mental health [10]. This fact is also evidenced in our study, being that despite having an SMI, none of our patients had a Disability Certificate.

Little is known about transgender womens' experiences accessing gender-segregated housing services, particularly services for homeless people. Only 
Table 1. Sociodemographic and clinical variables of homeless TW with SMI.

\begin{tabular}{|c|c|c|c|}
\hline & CASE 1 & CASE 2 & CASE 3 \\
\hline Year of birth & 1979 & 1972 & 1991 \\
\hline Nationality & Nigerian & Brazilian & Spanish \\
\hline Type of cohabitation prior to homelessness & $\begin{array}{c}\text { Family } \\
\text { cohabitation }\end{array}$ & $\begin{array}{c}\text { Family } \\
\text { cohabitation }\end{array}$ & $\begin{array}{c}\text { Institution } \\
\text { (Prison) }\end{array}$ \\
\hline Homelessness duration & Over 5 years & Over 5 years & Over 5 years \\
\hline Typology of homelessness & Houselessness & Rooflessness & Rooflessness \\
\hline Achieved educational level & Basic studies & Basic studies & Illiteracy \\
\hline Profession & $\begin{array}{c}\text { Eventual } \\
\text { non-qualified }\end{array}$ & $\begin{array}{c}\text { Eventual } \\
\text { non-qualified }\end{array}$ & $\begin{array}{c}\text { Without } \\
\text { known job }\end{array}$ \\
\hline Labor situation & Inactive & Inactive & Inactive \\
\hline Income sources & Does not have & Does not have & Does not have \\
\hline Health Coverage & Yes & No & Yes \\
\hline Disability Certificate & No & No & No \\
\hline Civil Incapacity & No & No & No \\
\hline Victim of VIOLENCE & Yes & Yes & Yes \\
\hline Diagnostic (ICD-10criteria) $^{*}$ & Schizophrenia & Schizophrenia & Schizophrenia \\
\hline Duration of Mental Illness & Over five years & Over five years & Over five years \\
\hline Prior contact with Mental Health & Yes & Yes & Yes \\
\hline Prior psychiatrichospitalization & Yes & Yes & Yes \\
\hline Severity of Mental Illness (CGI-S)** & Markedly ill & Markedly ill & Markedly ill \\
\hline Disease Awareness & Yes & No & No \\
\hline Attitude towards pharmacological treatment & Good & Good & $\mathrm{Bad}$ \\
\hline Substance abuse & No & No & Yes \\
\hline Comorbidity with HIV & Yes & No & No \\
\hline
\end{tabular}

${ }^{*}$ The ICD-10 classification of mental and behavioural disorders [7]. ${ }^{*}$ Clinical Global Impressions Severity Scale [8].

one of the three patients included in our study was housed in a gender-segregated shelter (the other two were in a situation of rooflessness). A recent study shows that exclusion from women-specific services had potentially severe adverse consequences such as homelessness and sexual violence [11]. And in this sense, all our patients had been victims of crimes while they were in a situation of homelessness.

Two of the three patients included in our study were migrants. Stigma and discrimination harm the wellbeing of TW and contribute to migration from contexts of sexual persecution and criminalization. Yet migrant TW often face marginalization and struggles meeting the social determinants of health following immigration to countries such as Spain. Social isolation is a key social determinant of mental health that may play a significant role in shaping health disparities among migrant TW [12]. Researchers are beginning to document the 
experiences of TW forced migrants in settlement. However, studies exploring the perceptions of service providers working with this vulnerable population are limited [13]. These may shed light on the challenges of meeting the needs of TW forced migrants, especially mental health issues and problems [14].

All our patients had been diagnosed with schizophrenia. In recent studies investigating the prevalence of psychiatric disorders among transgender people, major psychiatric disorders, such as schizophrenia, were rare and were no more prevalent than in the general population. Although many studies were methodologically weak, and included people at different stages of transition within the same cohort of patients, overall a recent review indicates that TW attending transgender health-care services appear to have a higher risk of psychiatric morbidity (that improves following treatment), and thus confirms the vulnerability of this population. Controversy exists as to if, and when, gender affirmative treatment should be offered to TW and co-existing psychosis. Concerns exist regarding regret afterward due to impulsive decision making, and deterioration of psychotic symptoms, but a recent study shows that gender affirmative treatment is possible and safe in this vulnerable population [15].

Finally, only one of our patients had HIV infection. The discrimination and stigma that TW with SMI encounter shape HIV vulnerability. For example, condomless sex has been found to be associated with unstable housing and experiences of transphobia among TW [11]. The potential costs of excluding TW from health and housing services include increased HIV vulnerability through reduced access to HIV prevention services, and through homelessness and social exclusion that are structural risk factors for HIV [11] [12].

\section{Conclusions}

Little is known about TW with SMI, but we know that they suffer significant levels of discrimination, stigma, and physical violence. This stigma plays an important role in limiting the opportunities and access to resources in a number of critical domains (e.g., medical care, disability certificate, accommodation in shelters) while continuously having a detrimental effect on their mental health. Forced migration in TW with SMI, must be specially considered. In addition homelessness and social exclusion are structural risk factors for HIV in TW with SMI. Social, health and psychiatric care may moderate the effect of stressors on mental health, reduce social isolation, and build social networks.

Small sample size and retrospective nature of data are the obvious limitations of the present study, and prospective studies with a large sample are required.

\section{Funding}

This study was carried out with its own resources and had no external funding.

\section{Conflicts of Interest}

The authors declare the absence of conflict of interests. 


\section{References}

[1] McCann, E. and Sharek, D. (2016) Mental Health Needs of People Who Identify as Transgender: A Review of the Literature. Archives of Psychiatric Nursing, 30, 280-285. https://doi.org/10.1016/j.apnu.2015.07.003

[2] Dhejne, C., Van Vlerken, R., Heylens, G. and Arcelus, J. (2016) Mental Health and Gender Dysphoria: A Review of the Literature. International Review of Psychiatry, 28, 44-57. https://doi.org/10.3109/09540261.2015.1115753

[3] Fernández García-Andrade, R., Serván, B., Medina, E., Vidal, V., Bravo, M. and Reneses, B. (2018) Criminal Behavior among Homeless Individuals with Severe Mental Illness. Revista Española de Medicina Legal, 44, 55-63. https://doi.org/10.1016/j.remle.2017.09.001

[4] Fernández García-Andrade, R., Serván, B., Reneses, B., Vidal, V., Medina, E. and Fernández, E. (2019) Forensic-Psychiatric Assessment of the Risk of Terrorist Radicalisation in the Mentally Ill Patient. Revista Española de Medicina Legal, 45, 59-66. https://doi.org/10.1016/j.remle.2019.01.003

[5] Ruggeri, M., Leese, M., Thornicroft, G., Bisoffi, G. and Tansella, M. (2000) Definition and Prevalence of Severe and Persistent Mental Illness. The British Journal of Psychiatry, 177, 149-155. https://doi.org/10.1192/bjp.177.2.149

[6] Federation of National Organisations Working with the Homeless (2012) On the Way Home? FEANTSA Monitoring Report on Homelessness and Homeless Policies in Europe. FEANTSA, Brussels.

[7] World Health Organization (2003) The ICD-10 Classification of Mental and Behavioural Disorders: Clinical Descriptions and Diagnostic Guidelines. World Health Organization, Washington DC.

[8] Guy, W. (1976) Clinical Global Impressions. In: ECDEU Assessment Manual for Psychopharmacology, National Institute of Mental Health, Rockville, 217-222. https://doi.org/10.1037/e591322011-001

[9] Feldman, B.J., Craen, A.M., Enyart, J., Batchelor, T., Friel, T.J., Dusza, S.W. and Greenberg, M.R. (2018) Prevalence of Homelessness by Gender in an Emergency Department Population in Pennsylvania. The Journal of the American Osteopathic Association, 118, 85-91. https://doi.org/10.7556/jaoa.2018.023

[10] Fernández-Rouco, N., Carcedo, R.J., López, F. and Orgaz, M.B. (2019) Mental Health and Proximal Stressors in Transgender Men and Women. Journal of Clinical Medicine, 8, 413. https://doi.org/10.3390/jcm8030413

[11] Lyons, T., Krüsi, A., Pierre, L., Smith, A., Small, W. and Shannon, K. (2016) Experiences of Trans Women and Two-Spirit Persons Accessing Women-Specific Health and Housing Services in a Downtown Neighborhood of Vancouver, Canada. LGBT Health, 3, 373-378. https://doi.org/10.1089/lgbt.2016.0060

[12] Logie, C.H., Lacombe-Duncan, A., Lee-Foon, N., Ryan, S. and Ramsay, H. (2016) "It's for Us-Newcomers, LGBTQ Persons, and HIV-Positive Persons. You Feel Free to Be": A Qualitative Study Exploring Social Support Group Participation among African and Caribbean Lesbian, Gay, Bisexual and Transgender Newcomers and Refugees in Toronto, Canada. BMC International Health and Human Rights, 16, 18. https://doi.org/10.1186/s12914-016-0092-0

[13] Gaboardi, M., et al. (2019) Goals and Principles of Providers Working with People Experiencing Homelessness: A Comparison between Housing First and Traditional Staircase Services in Eight European Countries. International Journal of Environmental Research and Public Health, 16, 1590. 
https://doi.org/10.3390/ijerph16091590

[14] Kahn, S., Alessi, E., Woolner, L., Kim, H. and Olivieri, C. (2017) Promoting the Wellbeing of Lesbian, Gay, Bisexual and Transgender Forced Migrants in Canada: Providers' Perspectives. Culture, Health \& Sexuality, 19, 1165-1179.

https://doi.org/10.1080/13691058.2017.1298843

[15] Meijer, J.H., Eeckhout, G.M., van Vlerken, R.H. and de Vries, A.L. (2017) Gender Dysphoria and Co-Existing Psychosis: Review and Four Case Examples of Successful Gender Affirmative Treatment. LGBT Health, 4, 106-114.

https://doi.org/10.1089/lgbt.2016.0133 